\title{
PATHOLOGICAL FRACTURE TIBIA DUE TO METASTATIC LESION FROM MALIGNANT MELANOMA OF FOOT: A CASE REPORT
}

Kunal R. Bansal11, Ajay Chandanwale 2

${ }_{1}^{1}$ Assistant Professor, Department of Orthopaedics, BJ Medical College, Pune.

${ }^{2}$ Dean and Professor, Department of Orthopaedics, BJ Medical College, Pune.

\section{ABSTRACT}

There is considerable variation in the reported incidence of skeletal metastasis from malignant melanoma. Metastasis to axial skeleton is almost four times more common than to appendicular skeleton. ${ }^{1}$ In long bones, the metastasis is usually located symmetrically at diaphysis. ${ }^{1}$ Patients with skeletal metastasis have grave prognosis with mean survival of 3.6 months. ${ }^{2}$ We report a case of malignant melanoma of foot with symmetrical metastasis to tibial diaphysis and pathological fracture on one side with 18 months' survival.

\section{KEYWORDS}

Pathological Fracture, Tibia, Malignant Melanoma of Foot.

HOW TO CITE THIS ARTICLE: Bansal KR, Chandanwale A. Pathological fracture tibia due to metastatic lesion from malignant melanoma of foot: a case report. J. Evolution Med. Dent. Sci. 2016;5(20):1067-1070, DOI: 10.14260/jemds/2016/247

\section{INTRODUCTION}

Malignant melanoma has an unpredictable clinical course in terms of metastatic behavior. ${ }^{3}$ There is considerable variation in the reported incidence of skeletal metastasis from malignant melanoma. Reported metastasis to axial skeleton is $80 \%$ and $20 \%$ to appendicular skeleton. In the long bones, metastasis is usually located symmetrically in the diaphysis with pathological fracture rate of $58 \%$ and usually associated with soft tissue swelling. ${ }^{1}$ Metastatic lesions are usually lytic on radiographs. We report a case of malignant melanoma of foot with symmetrical to tibial diaphysis and pathological fracture on one side.

\section{CASE REPORT}

In August 2010, a 62 years old female presented with pain in the middle of leg and multiple swellings in right lower limb since 12 months.

First swelling appeared on middle of leg, second on the lateral aspect of ankle and third in inguinal region, 6 months after appearance of swelling on the middle of leg, excision biopsy of the lesion was done followed by split skin grafting for closure of defect. Patient had trivial trauma 3 months back, sustained compound Grade 2 fracture tibia middle third.

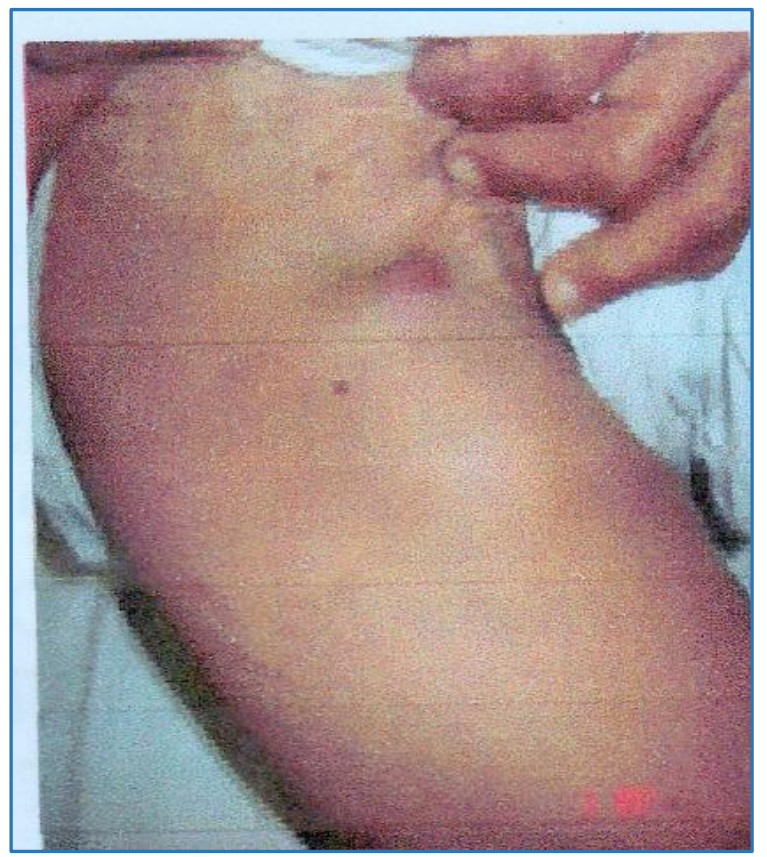

Fig. 1: Clinical Photograph of Swelling Over Thigh

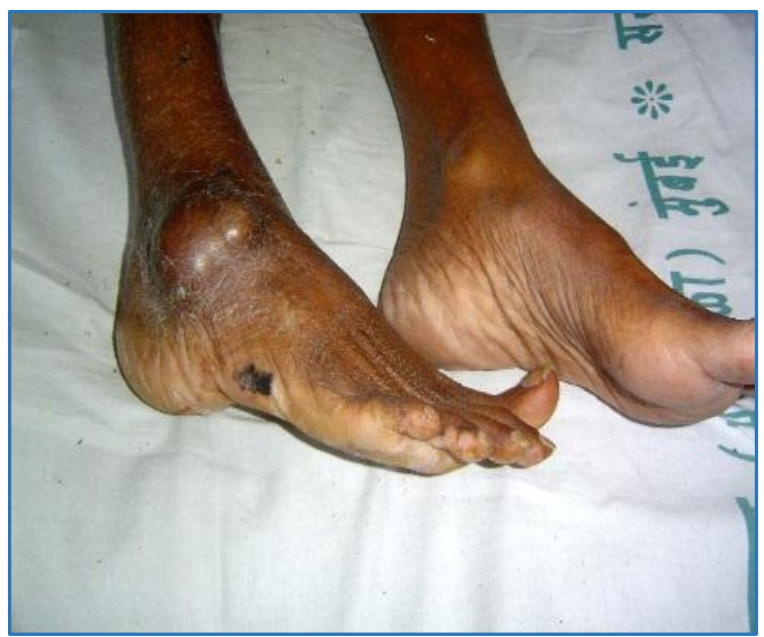

Fig. 2: Clinical Photograph of Swelling Over Ankle

Financial or Other, Competing Interest: None.

Submission 27-01-2016, Peer Review 21-02-2016,

Acceptance 27-02-2016, Published 10-03-2016.

Corresponding Author:

Dr. Kunal R. Bansal,

A-503, Ganga Melrose Sopan Baug,

Pune-411001.

E-mail:kun_bansal@yahoo.co.in

DOI: $10.14260 /$ jemds $/ 2016 / 247$ 


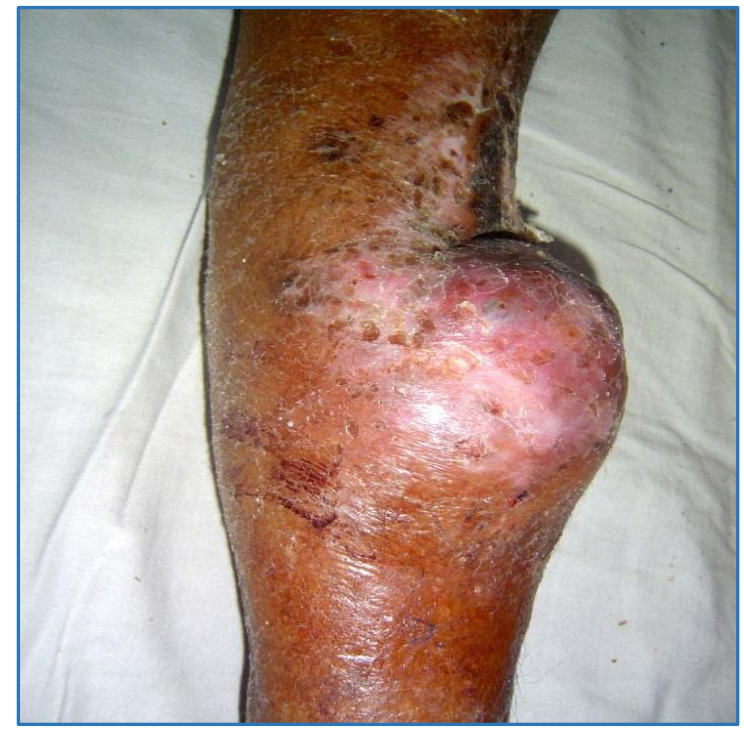

Fig. 3: Clinical Photograph of Lesion Over Tibia

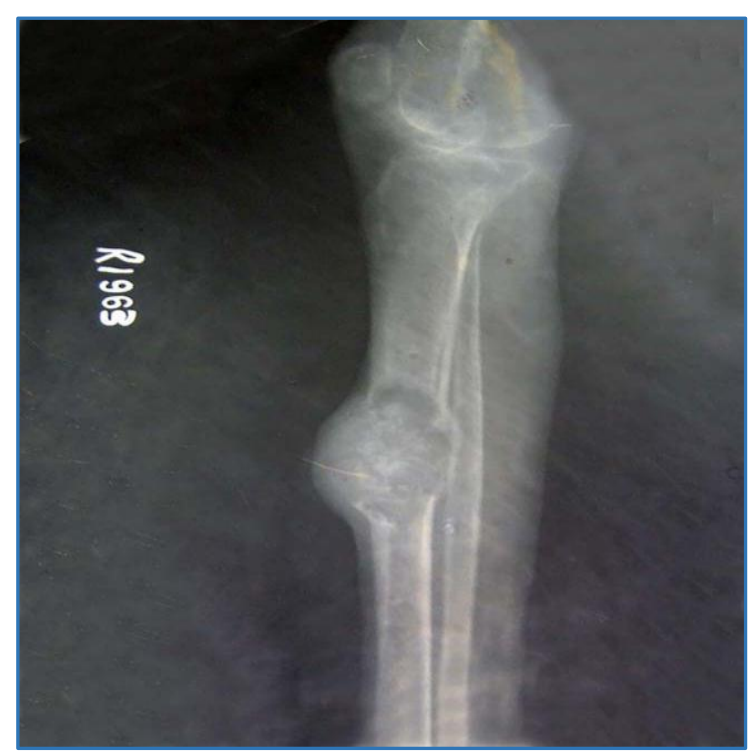

Fig. 4: X-ray showing Lytic Lesion Over Shaft Tibia

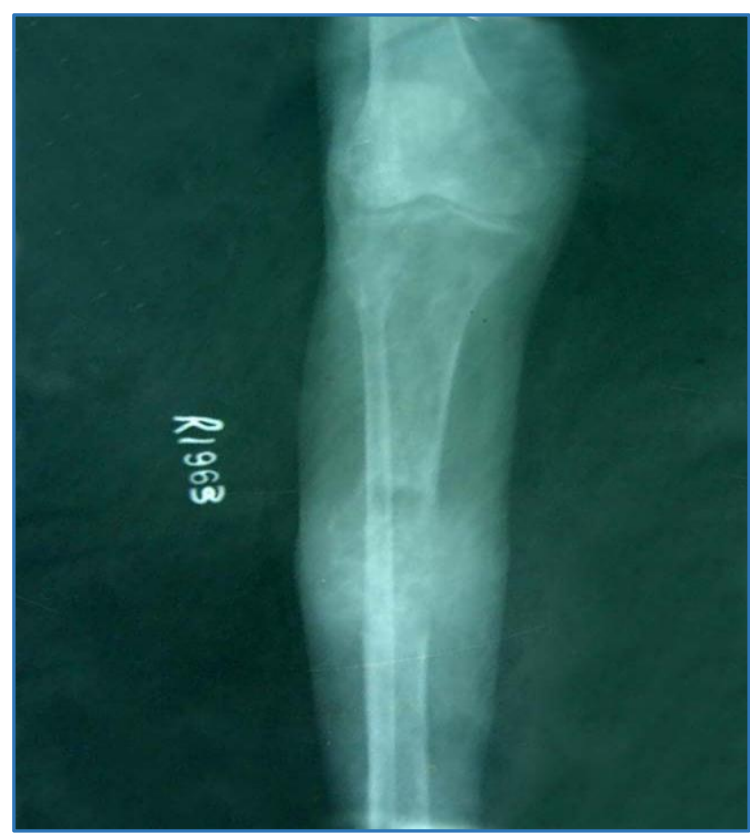

Fig. 5: X-ray showing Extensive Lysis in Shaft of Tibia

\section{Examination Findings}

Patient presented to us with a swelling in middle of leg-8*7 $\mathrm{cm}$, mobile, tender.

Tenderness and mobility in antero-posterior and lateral planes at the site of swelling in the leg. Swelling on right groin $-5^{*} 4 \mathrm{~cm}$ which was tender, hard, non-mobile. Swelling on right ankle $-5 * 4 \mathrm{~cm}$, hard, mobile and non-tender.

\section{INVESTIGATIONS}

Haematology - all values within normal limits, Serologynegative.

\section{A Series of Biopsies was done which are mentioned as follows:}

First biopsy from swelling over the leg showed evidence of histiocytoid haemangioendothelioma. Second J needle biopsy from swelling over the leg-Poorly differentiated malignant tumour with areas of necrosis. ImmunocytochemistryTumour cells showed discrete cytoplasmic and focal nuclear positivity for S100, HMB-45 negative. Melan A showed discrete, focal and weak cytoplasmic reactivity, CK and EMA (Epithelial marker) negative.

FNAC of right inguinal lymph node- Metastatic carcinoma cells, poorly differentiated malignant tumour with possibility of melanoma.

USG of right leg $7.8^{*} 6.9 \mathrm{~cm}$ swelling. Increased vascularity shows neoplastic rather than infection. Arterial and venous Doppler of both legs-Normal CT ThoraxSuggested features of old tuberculosis.

MRI right leg-Large, lobulated, expansile, destructive lesion in mid diaphysis of right tibia, similar lesion over lateral malleolus. Impression-Multicentric benign vascular neoplasm.
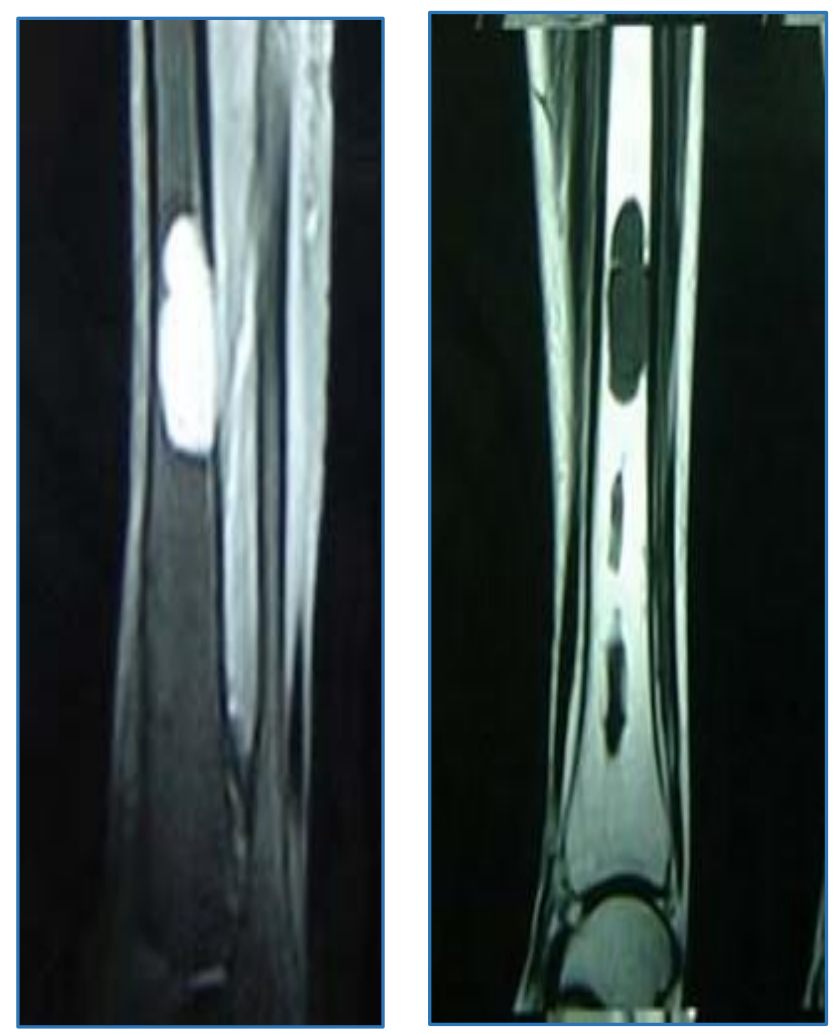

Fig. 6: MRI Left Leg-Multiple well-defined Intramedullary Lesions in the Distal Metaphysic of the tibia with narrow zone of transition, findings suggestive Malignant/Metastatic Lesion 


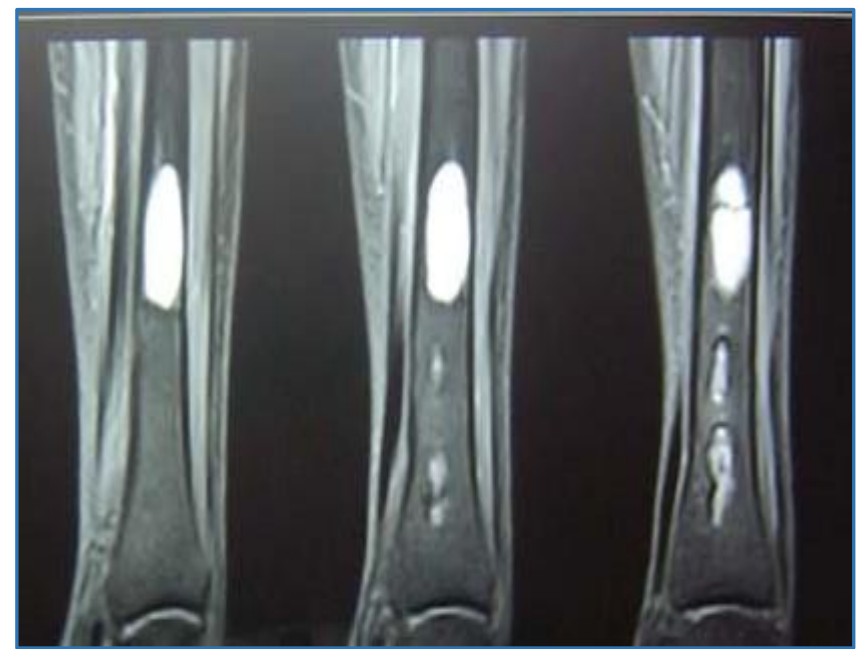

Fig. 7: Bone Scan-Increased Radiotracer uptake seen in the Midshaft of Right and Left Tibia

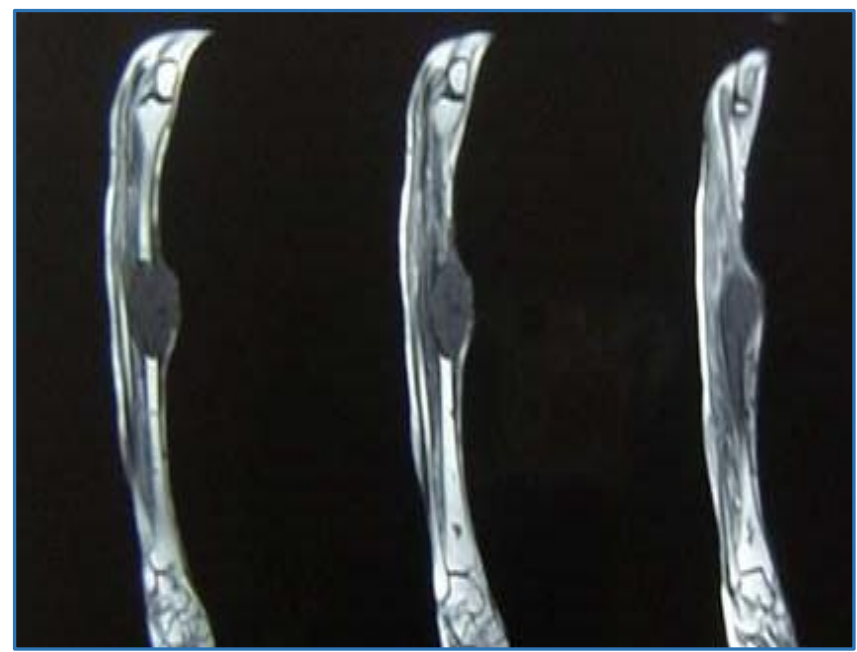

Fig. 8: Bone Scan-Increased Radiotracer uptake seen in the Midshaft of Right and Left Tibia

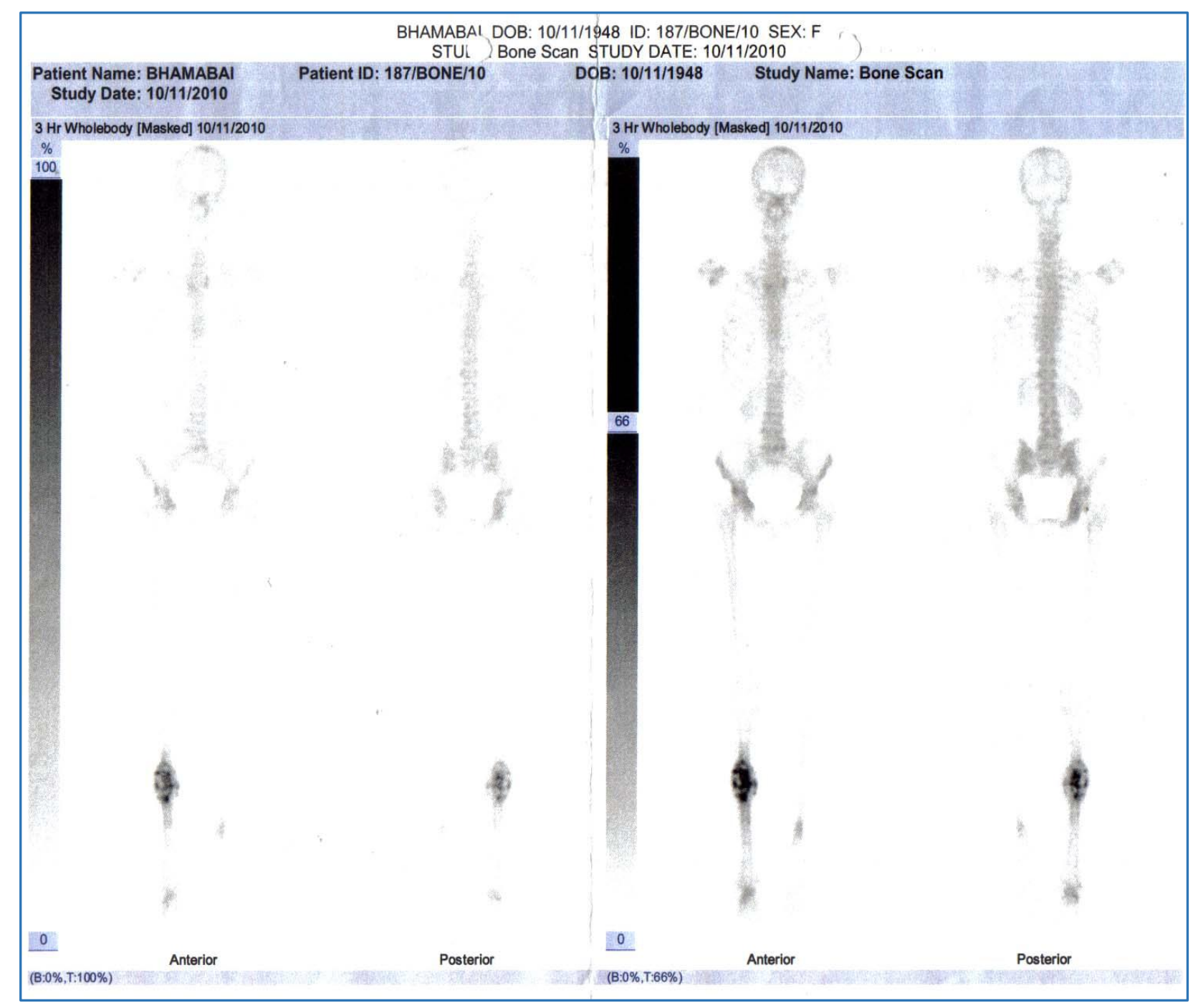

Fig. 9: PET Scan-Increased tracer uptake in Right Midshaft Tibia, Right Foot, Right Inguinal Region Represents Metabolically Active Metastatic Lesion 


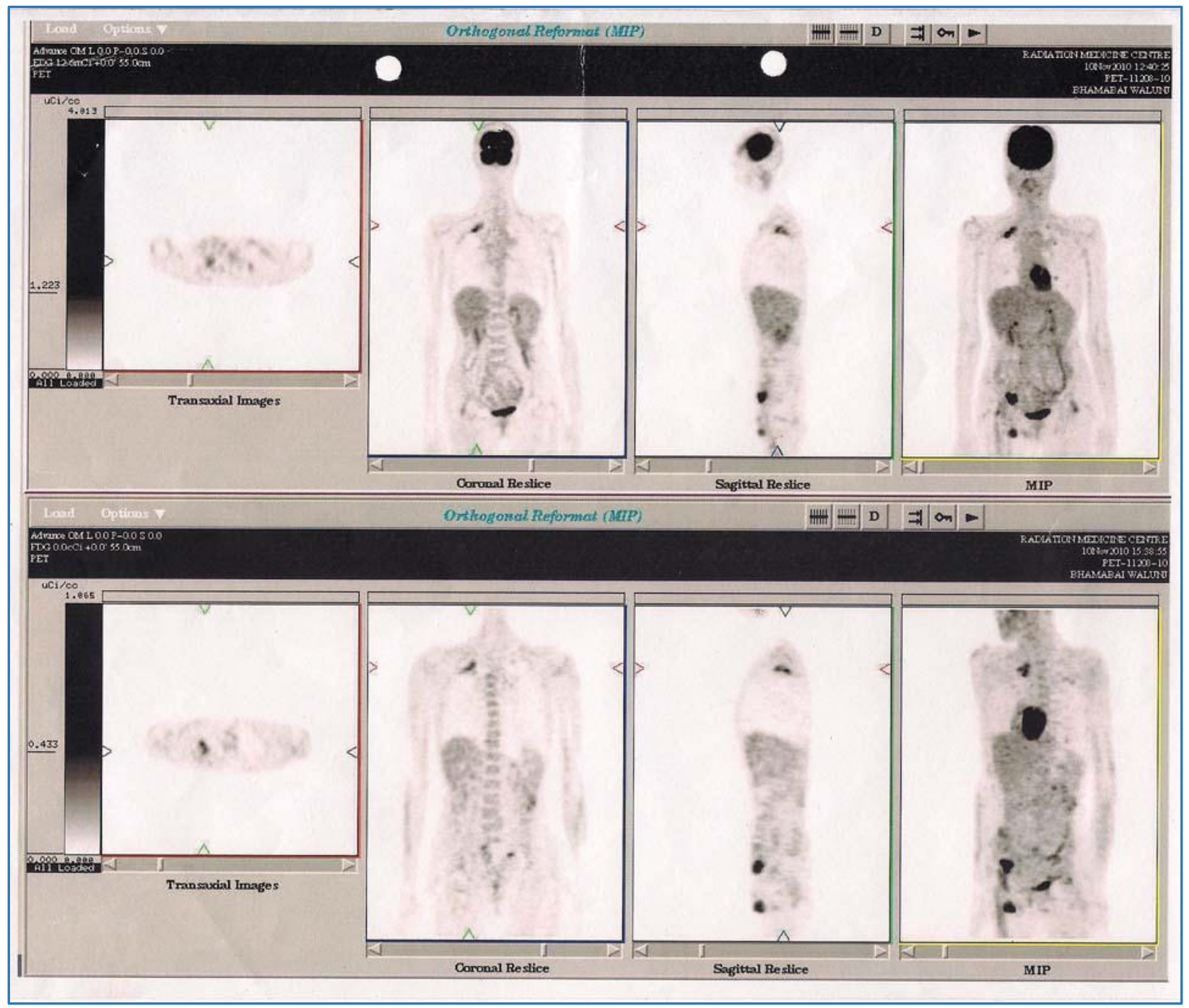

Fig. 10: PET Scan-Increased tracer uptake in Lungs, Inguinal Region, Pelvic Bone Represents Metabolically Active Metastatic Lesion

\section{DISCUSSION}

The case reported here is considered initially like a soft tissue swelling over the leg treated with incisional biopsy, which showed features of Histiocytoid haemangioendothelioma. Later on following trivial trauma, patient sustained pathological fracture of tibia and radiograph showed lytic lesion at fracture site. Repeat biopsy was done, which showed features of malignant tumour and attention was drawn towards hyperpigmented (Black) patch over the lateral aspect of foot, which was malignant melanoma. Patient had no pain on left-sided tibia, although bone scan and PET scan showed similar lesions as on right, but of milder severity. The skeletal metastasis weakens the bone considerably resulting into pathological fracture, as the reported incidence of pathological fractures Id's $58 \% .^{1}$ The soft tissue swelling is usually seen in association with the fracture.

Primary cutaneous melanoma has been reported to be the most common malignant lesion affecting the foot. ${ }^{4}$ The term acral lentiginous melanoma refers to a melanoma occurring on the hand and the foot. It is more common in females.(2,3,1) Plantar aspect being the most common site. ${ }^{4}$
Patients with skeletal metastasis have a grave prognosis with the mean survival of 3.6 months. ${ }^{2}$

The bone scan helped in detecting similar metastatic lesions in the bones, whereas PET scan detected metastasis to other systems. The reported case has bilateral tibial, right ankle joint and right inguinal lymph node involvement with no visceral metastasis and survival period of more than 18 months.

\section{REFERENCES}

1. Gerald T Fon, Wong WS, Gold RH, et al. Skeletal metastasis of melanoma: radiographic scintigraphic and clinical review. AJR 1981;137(1):103-108.

2. Stewart WR, Gelberman Rh, Harrelson Jm, et al. Skeletal metastasis of melanoma. J Bone Joint Surg 1978;60(5):645-649.

3. de la Monte SM, Moore GW, Hutchins GM. Patterned distribution of metastasis from malignant melanoma in humans. Cancer research 1983;43(7):3427-3433.

4. Booher RJ, Pack GT. Malignant Melanoma of the feet and hands. Surgery 1957;42(6):1084-1121. 